\title{
ASYMPTOTIC SOLUTIONS FOR SHELLS WITH GENERAL BOUNDARY CURVES*
}

\author{
BY \\ T. R. LOGAN \\ McDonnell Douglas Corporation, Long Beach, California
}

\begin{abstract}
The influence of arbitrary edge loads on the stresses and deformations of thin, elastic shells with general boundaries is studied by means of asymptotic expansions of a general tensor equation. Expansions are made in terms of an exponential or an Airy function and a series in powers of a small-thickness parameter. Most of the steps in the procedure are effected by using the dyadic form of the tensors. Solutions are obtained that are valid in the large, with no restrictions on the loading or on the boundary geometry.

Results indicate that the behavior of shells with arbitrary boundary geometry can be quite different from that in the ordinary case. Specific results show the presence of an interior caustic which is the envelope of the characteristics of an eiconal equation. The exponential expansion becomes singular at the caustic, which would generally be expected to be a local region of stress concentration.

The results have a close identity with asymptotic solutions obtained in geometric optics. Following some new techniques used for solution of the reduced wave equation, a solution of the shell equation is obtained using an asymptotic expansion in terms of Airy functions which provides a solution that is uniformly valid in the neighborhood of the caustic.

Introduction. The primary purpose of this study is to investigate the influence of general edge loads on the stresses and deformations of thin elastic shells. The shell equations were cast into a single, compact dyadic form by Steele [1] that will be used here without development. Some details of that derivation and additional algebraic details of equations developed in this paper are given in [2]. The type of solution sought is a generalization of the well-known solution, with a decaying behavior from the shell boundary into the shell interior, commonly referred to as an edge-effect solution.

Analytic solutions available in the literature are applicable only to shells with restricted boundaries and loading conditions, or are valid only close to the boundary. Certainly the most widely used edge-effect solution is that for the axisymmetricallyloaded circular cylinder with a boundary on a circumferential line of curvature, and that result may be obtained as a special case of our general solution.

Perhaps the best known solution for a shell with an arbitrary boundary is that of Goldenveizer [3]. However, that solution involves a basic assumption that restricts the region of applicability of the solution to a small boundary layer near the shell edge.
\end{abstract}

- Received October 10, 1971. This study, performed at Stanford University, was supported by the McDonnell Douglas Corporation and by the National Science Foundation under Grant GK-2701. 
In particular, for the "simple edge-effect solution," it is assumed that the state of stress increases by an order of the reciprocal of the square-root of the thickness when differentiated along directions normal to the boundary, but has negligible change when differentiated along the boundary. In addition, it is assumed that the geometric properties are essentially constant in directions normal to the boundary. These basic assumptions yield a simple solution that resembles the usual solution for cylinders, but in terms of a local normal curvature. The assumptions necessarily limit the range of validity of the solution to a region near the edge, and to loading that has a slow variation along the edge.

The problem of a circular cylinder with an oblique edge cut-off is solved by van der Neut [4] by approximating the edge with a helix, and assuming slow variations in loading and geometry. More recently, Kitching and Bond [5] have solved the problem of the intersection of pipes at oblique angles. Their edge-effect solution corresponds to the one described above, due to Goldenveizer, and uses a solution in terms of a local normal curvature. The problem of the oblique intersection of a sphere with a circular cylinder is solved by Johnson [6]. That solution uses the assumption, among some others, of the applicability of the edge-effect solution for a cylinder with the boundary on a circumferential line of curvature.

In addition to problems of the type discussed above, the problem of a shell with a boundary that is tangent at discrete points to asymptotic lines of the shell surface is of particular interest. The problem is typified by a cylinder with a cutout, since in a cylinder the generators are asymptotic lines. Naghdi and Eringen [7] solve the problem by assuming flat plate behavior in the region of interest, so that the solution is restricted to small openings. A similar restriction also limits the applicability of solutions of Savin and Guz [8], Lure [9], and, more recently, Van Dyke [10]. Recently, an extensive series solution for an elliptical hole in a cylindrical shell has been used by Rao and Ariman [11]. All of these solutions have important practical value, but involve assumptions that limit the results to spacial circumstances.

In this study we consider arbitrary shells and seek solutions that are uniformly valid in the large, without restriction to specific loading or boundary shape. However, the difficulty in this analysis is the solution of a first-order, nonlinear differential equation in the large, and to date closed-form solutions have been obtained only for special cases.

In Sec. I the differential equations are summarized, and in Sec. II an exponential expansion is detailed. In Sec. III, the details of an Airy function expansion are presented and the results are compared to those obtained from the exponential expansion. Sec. IV presents some elementary cases and illustrates the application of our solutions.

I. Governing differential equation. In this section the governing differential equation is summarized. The historical background and a careful development of the foundations of thin shell theory is given by Naghdi [12]. For solving problems, there are many advantages in using the fourth-order system of partial differential equations in terms of complex dependent variables. Using the dyadic representation of tensors, rather than the component form, this system of complex equations was written as a single equation by Steele [1]. In general, we consider tensors of rank two, and repeated indices are summed over the values 1,2 , unless otherwise noted. A tensor of rank two is indicated by the notation $\mathbf{T}=T^{\alpha \beta} \mathbf{a}_{\alpha} \otimes \mathrm{a}_{\beta}$, where $\mathbf{a}_{\alpha}$ are the base vectors at the point and $\otimes$ indicates the tensor product. 
The homogeneous form of the governing differential equation is written as

$$
\nabla \cdot\left[\hat{\mathbf{P}}+i c(\nabla \hat{\mathbf{P}}) \otimes \mathbf{a}_{3}\right]=0 .
$$

The tensor $\hat{\mathbf{P}}$ is a symmetric form whose components contain equilibrium stress resultants and couples as the real parts and strains and curvatures as the imaginary parts. The variable $\hat{P}$ is the trace of $\hat{\mathbf{P}}$ and this correspondence in notation is used throughout the paper. The unit normal vector to the surface is $\mathbf{a}_{3}$ and $c$ is a reduced thickness given by $c=h /\left[12\left(1-\nu^{2}\right)\right]^{1 / 2}$. Finally, the operator $\nabla$ is the usual gradient operator.

The components of Eq. (1.1) give the equations obtained by Naghdi [13], which for lines-of-curvature coordinates, reduce to the form of the equations of Novozhilov [14], but with the modified stress resultants and the curvature measure due to Sanders [15] and Koiter [16]. Eq. (1.1) is a vector equation which when expanded gives a system of partial differential equations containing two first-order equations and one secondorder equation. The system is equivalent to a single eighth-order equation, and contains equilibrium equations as real parts and compatibility conditions as imaginary parts, but connected by constitutive relations.

II. Exponential solutions. In this section we consider a solution of Eq. (1.1) that exhibits exponential behavior. Generally, this results in the "edge-effect" or "boundarylayer" type of solution that decays exponentially from the shell boundary into the interior. However, the influence of self-equilibrating edge loads can often be significant far into the shell interior, for example in shells of negative and zero curvature. Here we seek a solution that is uniformly valid in the large, without a priori assumptions regarding behavior. One way that the solution has been obtained in the literature, for the special case of boundaries that are lines-of-curvature, is by the use of an asymptotic expansion in terms of the small thickness parameter. Motivated by these known solutions, the procedure for the case of arbitrary boundaries will be to seek a formal asymptotic solution for $\hat{\mathbf{P}}$ in terms of an exponential and a series of tensors in powers of the small-thickness parameter.

Asymptotic expansion. It is convenient to write Eq. (1.1) in the form

$$
\nabla \cdot \hat{\mathbf{P}}+\lambda^{-2}\left(\Delta \hat{P} \mathrm{a}_{3}-\nabla \hat{P} \cdot \mathbf{b}\right)=0
$$

where we have introduced the notation $\lambda=(i c)^{-1 / 2}$. The components of the tensor $\mathbf{b}$ are the coefficients of the second fundamental form and $(\triangle)$ is the Laplacian operator.

We assume solutions of Eq. (1.1) of the form

$$
\hat{\mathbf{P}}=\exp \left[\lambda \xi\left(x^{1}, x^{2}\right)\right] \mathbf{P}
$$

where

$$
\mathbf{P}=\sum_{k=0}^{n} \lambda^{-k} \mathbf{P}_{(k)}
$$

in which each $\mathbf{P}_{(k)}$ is independent of $\lambda$. Substituting Eq. (2.2) into Eq. (2.1) and equating the coefficient of each power of $\lambda$ to zero, we obtain the general equation

$$
\begin{aligned}
\nabla \xi \cdot \mathbf{P}_{(k)}+\nabla \cdot \mathbf{P}_{(k-1)}+\nabla \xi \cdot \nabla \xi P_{(k-1)} \mathrm{a}_{3}+2 \nabla \xi \cdot \nabla P_{(k-2)} \mathbf{a}_{3} \\
\quad+\triangle \xi P_{(k-2)} \mathbf{a}_{3}-P_{(k-2)} \nabla \xi \cdot \mathrm{b}+\triangle P_{(k-3)} \mathbf{a}_{3}-\nabla P_{(k-3)} \cdot \mathrm{b}=0
\end{aligned}
$$

where $\mathbf{P}_{(i)}=0$ for all $j<0$. Eq. (2.4) may now be systematically solved for sequential 
values of the index. For further solution, it is convenient to separate the equation into normal and tengential parts which are, respectively,

$$
\begin{gathered}
\mathbf{a}^{\lambda} \cdot \mathbf{P}_{(k-1)} \cdot \mathbf{b} \cdot \mathbf{a}_{\lambda}+\nabla \xi \cdot \nabla \xi P_{(k-1)}+2 \nabla \xi \cdot \nabla P_{(k-2)} \\
+\triangle \xi P_{(k-2)}+\Delta P_{(k-3)}=0, \\
\nabla \xi \cdot \mathbf{P}_{(k)}+\left.P_{(k-1)}^{\alpha \beta}\right|_{\alpha} \mathbf{a}_{\beta}-P_{(k-2)} \nabla \xi \cdot \mathbf{b}-\nabla P_{(k-3)} \cdot \mathbf{b}=0 .
\end{gathered}
$$

Eiconal function. The first equation to be considered is obtained from Eq. (2.6) as

$$
\nabla \xi \cdot \mathbf{P}_{(0)}=0 .
$$

A solution of Eq. (2.7) may be written in the form $\mathbf{P}_{(0)}=f_{\varepsilon} \cdot \nabla \xi \otimes \nabla \xi \cdot \varepsilon$, where $f$ is an undetermined scalar function. The permutation tensor $\varepsilon$ operates with the scalar product on the intrinsic part of a vector in the prefactor position to produce a rightangle counter-clockwise rotation, and in the postfactor position to produce a right-angle clockwise rotation, both rotations without change of length.

The tensor $\hat{\mathbf{P}}$ was defined to be symmetric and we therefore seek those $\mathbf{P}_{(k)}$ which are themselves symmetric. To determine $f$, both sides of the equation above are contracted by replacing the tensor product with the scalar product to yield the solution

$$
\mathbf{P}_{(0)}=-P_{(0)} \mathbf{\Psi} \text {. }
$$

We have defined $P_{(0)}=$ trace $\mathbf{P}_{(0)}$, and

$$
\mathbf{\Psi}=\frac{\varepsilon \cdot \nabla \xi \otimes \nabla \xi \cdot \varepsilon}{\nabla \xi \cdot \nabla \xi} .
$$

The solution of Eq. (2.8) is now substituted into Eq. (2.5) with an index value of 1 . Thus we obtain the governing equation for $\xi$ as

$$
\nabla \xi \cdot \varepsilon \cdot \mathbf{b} \cdot \varepsilon \cdot \nabla \xi-(\nabla \xi \cdot \nabla \xi)^{2}=0 .
$$

As the solutions are developed, certain analogies with well-known asymptotic solutions of the reduced wave equation will appear; see, for example, Lewis and Keller [17]. Since it is convenient to have names for some of the equations being used, we adopt the terminology of geometric optics, so that Eq. (2.10) will be referred to as the eiconal equation, with $\xi\left(x^{1}, x^{2}\right)$ the eiconal or phase function.

Transport equations. The remaining normal equations can be cast into the general form

$$
\nabla \xi \cdot \mathbf{P}_{(\lambda)}+\mathbf{u}_{(\lambda)}=0 .
$$

Eq. (2.11) is linear and the solution may be represented as the sum of a homogeneous part and a particular part. The homogeneous solution is obtained as above in the form

$$
\mathbf{P}_{(\lambda) H}=-P_{(\lambda)} \mathbf{\Psi} .
$$

A symmetric particular solution is obtained as

$$
\mathbf{P}_{(\lambda) P}=-\mathbf{L} \otimes\left[\mathbf{u}_{(\lambda)}\right],
$$

where we have defined the linear operator $L$ such that

$$
\mathbf{L} \otimes[\mathbf{v}]=\frac{\nabla \boldsymbol{\xi} \otimes \mathbf{v}+\mathbf{v} \otimes \nabla \boldsymbol{\xi}-(\nabla \boldsymbol{\xi} \cdot \mathbf{v}) \boldsymbol{\delta}}{\nabla \xi \cdot \nabla \boldsymbol{\xi}} .
$$


The identity tensor $\boldsymbol{\delta}$ is defined by the relation $\delta=a_{\alpha \beta} \mathrm{a}^{\alpha} \otimes \mathrm{a}^{\beta}$, where the functions $a_{\alpha \beta}$ are the coefficients of the first fundamental form. The scalar product of $\boldsymbol{\delta}$ with a vector in the prefactor or postfactor position gives the intrinsic part of the vector.

The governing differential equation for $P_{(0)}$ is now obtained by substituting Eqs. (2.8) and (2.10) into Eq. (2.5) for an index value of 1 . The equation in its final form becomes

$$
\nabla P_{(0)} \cdot \mathbf{\Lambda}+P_{(0)} \Gamma=0,
$$

where the following definitions have been used:

$$
\begin{aligned}
\mathbf{\Lambda} & =2 \nabla \xi+\mathbf{\Psi} \cdot \mathbf{X}, \\
\Gamma & =\Delta \xi+\nabla \cdot \Psi \cdot \mathbf{X}, \\
\mathbf{X} & =X_{\beta} \mathbf{a}^{\beta} .
\end{aligned}
$$

The components of $X$ are given by $X_{\beta}=\mathrm{a}^{\lambda} \cdot \mathbf{L} \otimes\left[\mathbf{a}_{\beta}\right] \cdot \mathbf{b} \cdot \mathbf{a}_{\lambda}$. Following the previous remark, we will refer to Eq. (2.15) as the first transport equation, and to $P_{(0)}$ as the first transport or amplitude function.

Using the same procedures as above, we may now obtain all of the remaining transport equations in the same form as the first equation. The only modification needed is to account for the inhomogeneous part of the solution for the transport tensors with an index of two or more. The general transport equation may finally be obtained as

$$
\begin{aligned}
\nabla P_{(k)} \cdot \mathbf{\Lambda}+P_{(k)} \Gamma+\nabla \cdot \mathrm{V}_{(k)} \cdot \mathbf{X}+\Delta P_{(k-1)}-P_{(k-2)} \nabla \xi \cdot \mathbf{b} \cdot \mathbf{X} \\
\\
-\nabla P_{(k-3)} \cdot \mathbf{b} \cdot \mathbf{X}=0 .
\end{aligned}
$$

In addition to Eqs. (2.16) through (2.18) we have defined the tensor

$$
\mathbf{V}=V_{(\lambda)}^{\alpha \beta} \mathbf{a}_{\alpha} \otimes \mathbf{a}_{\beta},
$$

where

$$
V_{(\lambda)}^{\alpha \beta}=\mathbf{a}^{\alpha} \cdot \mathbf{L} \otimes\left[\mathbf{u}_{(\lambda)}\right] \cdot \mathbf{a}_{\beta}
$$

and

$$
\mathbf{u}_{(k)}=\left.P_{(k-1)}^{\alpha \beta}\right|_{\alpha} \mathbf{a}_{\beta}-P_{(k-2)} \nabla \xi \cdot \mathbf{b}-\nabla P_{(k-3)} \cdot \mathbf{b} .
$$

Eq. (2.19) is actually valid for all $k$ if terms with negative index in (2.21) are set equal to zero. All terms in the equation are known, theoretically, in terms of previous transport functions and the eiconal function. The solutions of the eiconal and transport equations will then give the complete asymptotic solution. From practical considerations, however, if satisfactory results are not obtained with the first few terms, then some approach other than this asymptotic expansion would be necessary.

Solution of the eiconal equation. Equation 2.10 is a first-order nonlinear partial differential equation for the eiconal function. For detailed solution, it is convenient to reduce the equations to their component form. We consider surface coordinate systems that are orthogonal, so that $a_{12}=a_{21}=0$. Using the notation $p=\partial \xi / \partial x^{1}$ and $q=$ $\partial \xi / \partial x^{2}$, Eq. (2.10) is written as

$$
p^{2} \frac{b_{22}}{a}+q^{2} \frac{b_{11}}{a}+\left(\frac{p^{2}}{a_{11}}+\frac{q^{2}}{a_{22}}\right)^{2}=0,
$$


where $a=\left|a_{\alpha \beta}\right|$ and the functions $b_{\alpha \beta}$ are the coefficients of the second fundamental form.

Eq. (2.22) is solved for particular shell configurations using the method of characteristics so that along characteristics solutions of the eiconal equation are obtained as solutions of ordinary differential equations. It is of particular interest to note that the characteristics are in general not parallel and so intersect at what will be termed focal points. The locus of these focal points is the envelope of the characteristics, and defines a caustic which is a line of singularities of solutions of the transport equations. Thus, at a caustic, the coefficient of $P_{(0)}$ in the first transport equation becomes unbounded so that a solution of the form of Eq. (2.1) is no longer valid. A demonstration of this singularity is given by Steele [18] and by Lewis et al. [19] for the reduced wave equation. We only note here that the Lamé parameter which measures the separation of the characteristics becomes zero at the caustic and since it appears in the denominator of the Laplacian of the eiconal function, $P$ becomes unbounded. In general, however, the eiconal function itself is not singular at the caustic.

For the shell problem, we interpret the caustic as an area of stress intensification. At interior points, the solution of the shell equation is ordinarily influenced by data specified on the point of the boundary that is intersected by the characteristic that passes through the interior point. However, points on the caustic are intersections of adjacent characteristics so that the solution will be influenced by data in a neighborhood of a boundary point. This increase in the normally expected value of the solution is obtained in Sec. IV for a special case.

A convenient specification of initial data is given by a parametric representation of the boundary edge in the form of functions $x^{1}(t)$ and $x^{2}(t)$, with $t$ the boundary parameter. To obtain the proper condition on the eiconal function, however, further considerations are necessary. For a periodic variation of continuous boundary data, the eiconal function may be written as $\xi=\zeta+i(n / \mu) \sigma$, where $\sigma$ is arc length along the edge, $\zeta$ is a function of the shell coordinates which is zero at the boundary, and the data have a wave length of $2 \pi \mu / n$. For axisymmetric deformations of shells of revolution, $n=\xi=0$ at the edge, and in the interior, $\xi^{-2}=O(h R)$. Now, in the development of the governing equation and its solution, terms of $O(h / R)$ compared to unity were neglected. It is then consistent to neglect contributions due to the variation of the boundary data that are of the same order of smallness when compared to $\zeta$. Expecting the same orderof-magnitude behavior for the general shell as for the shell of revolution, we define "slowly-varying" loads as those for which $\mu=O(R)$ and specify $\xi=0$ at the boundary. We define "rapidly-varying" loads as those for which $\mu<O(R)$ and specify $\xi=i(n / \mu) \sigma$ at the boundary. There is an upper limit on $n$, however, due to the neglect of transverse shear effects in the equation development; it is given by Steele [20] as $n \ll R / h$.

Solution of transport equations. First the solution of Eq. (2.15) will be considered. Steele [1] shows that $\boldsymbol{\Lambda}$ at each point is in the direction of the characteristics of the eiconal function. We introduce the parameter $\sigma_{1}$ as the coordinate parameter of the orthogonal trajectories of the characteristics. Then, using these coordinates, the first transport equation may be written as the ordinary differential equation

$$
\left(d P_{(0)} / d \sigma_{1}\right)+P_{(0)}(\Gamma / \Lambda)=0,
$$

where $\boldsymbol{\Lambda}=\Lambda \mathfrak{u}_{1}$ and $\mathfrak{u}_{1}$ is the base vector that corresponds to $\sigma_{1}$. 
Setting

$$
\gamma=\int^{\sigma_{1}} \frac{\Lambda}{\Gamma} d \sigma_{1}
$$

we write the solution of $(2.23)$ as

$$
P_{(0)}=P e^{-\gamma},
$$

where $P$ is the arbitrary integration factor. The solution for the first transport tensor is then

$$
\mathbf{P}_{(0)}=-P e^{-\gamma} \mathbf{\Psi}
$$

and is now determined in terms of the eiconal function. Although, as previously noted, transport functions after the first few are generally of little use for computations, it is of interest to develop their equations. Along characteristics, Eq. (2.19) becomes

$$
\left(d P_{(k)} / d \sigma_{1}\right)+P_{(k)}(\Gamma / \Lambda)=\Omega_{(k)},
$$

where

$$
\Omega_{(k)}=-\left(\nabla \cdot \mathrm{V}_{(k)} \cdot \mathrm{X}+\Delta P_{(k-1)}-P_{(k-2)} \nabla \xi \cdot \mathrm{b} \cdot \mathrm{X}-\nabla P_{(k-3)} \cdot \mathrm{b} \cdot \mathrm{X}\right) .
$$

The homogeneous solution is already included in the solution of the first transport equation, and so an additional integration factor would only change the arbitrary $P$. Then, the solution of Eq. (2.27) may be taken as

$$
P_{(k)}=e^{-\gamma} \int^{\sigma_{1}} \Omega_{(k)} e^{\gamma} d \sigma_{1},
$$

so that

$$
\mathbf{P}_{(k)}=-P_{(k)} \mathbf{\Psi}-\mathbf{L} \otimes\left[\mathbf{u}_{(k)}\right] .
$$

Eq. (2.29) is the solution of the general transport tensor. This solution may be written in terms of previous solutions by defining the operator $M$ such that $\Omega_{(k)}=M\left[P_{(k-1)}\right]$, where

$$
M\left[P_{(\lambda)}\right]=\left(\nabla \cdot \mathrm{V}_{(\lambda)} \cdot \mathrm{X}+\Delta P_{(\lambda-1)}-P_{(\lambda-1)} \nabla \xi \cdot \mathrm{b} \cdot \mathrm{X}-\nabla P_{(\lambda-3)} \cdot \mathrm{b} \cdot \mathrm{X}\right) / \Lambda .
$$

Finally we obtain the general result

$$
P_{(k)}=e^{-\gamma} \int^{\sigma_{1}} M\left[P_{(k-1)}\right] e^{\gamma} d \sigma_{1} \quad(k \geq 1) .
$$

The complete solution is now determined, in theory, as a sequence of integrals involving the eiconal function. Apparently the integrations will in general have to be performed numerically. However, the solution can be obtained in closed form for the special case of axisymmetric deformations of shells of revolution, for which the solution by other methods is known. The reduction of the eiconal equation for this special case is shown in Sec. IV.

In this section we have obtained an asymptotic solution of the thin-shell equations that is valid in the large except in a region near "caustics". Due to the existence of these caustics, the shell will exhibit behavior different from that usually obtained using the 
axisymmetric edge-effect solution. Some elementary examples in Sec. IV make this behavior more clear.

III. Airy function solutions. In this section we scek an asymptotic solution of the shell equation that will be valid for a region of the shell including the caustic. The motivation for the exponential expansion form was a straightforward extension of the usual expansion used for shells with boundaries that are on lines of curvature. It was necessary only to extend the expansion to a tensor form to suit our equation. The motivation for an expansion form that will give a uniformly valid solution through the caustic region is not quite so clear.

Considering the analogy to geometric optics, the Airy functions [21], developed to. describe light intensity at caustics, appear as a natural choice. The proper combination of terms can be developed from physical arguments, but a more compelling mathematical argument was developed by Ludwig [22]. He used an argument based on a superposition of plane-wave solutions as a solution of the reduced wave equation, and then used an extended steepest-descent method to develop an Airy-function expansion form that gave the desired results. We use the same expansion, making modifications only for the tensor notation and the fact that our expansion is in terms of a complex parameter. It remains to be shown in what follows that the phase angle of the Airy function argument is of the proper magnitude so that our asymptotic reductions will be valid. As will be seen, with the proper definition of variables, the Airy function solution may be handled in the same way as the exponcntial function solution, the only essential complication being an increase in the number of equations.

Asymptotic expansion. Solutions of Eq. (2.1) are assumed in the form

$$
\hat{\mathbf{P}}=e^{i \lambda \theta}\left[A_{i}\left(-\lambda^{2 / 3} \zeta\right) \mathbf{Q}+i \lambda^{-1 / 3} A_{i}^{\prime}\left(-\lambda^{2 / 3} \zeta\right) \mathrm{R}\right],
$$

where

$$
\begin{aligned}
& \mathbf{Q}=\sum_{k=0}^{n} \lambda^{-k} \mathbf{Q}_{(k)}, \\
& \mathbf{R}=\sum_{k=0}^{n} \lambda^{-k} \mathbf{R}_{(k)},
\end{aligned}
$$

and where $\theta$ and $\zeta$ are functions of the shell coordinates. Each $\mathbf{Q}_{(k)}$ and $\mathbf{R}_{(k)}$ is independent of $\lambda$.

To obtain a formal solution, we substitute Eq. (3.1) into Eq. (1.1) and collect terms by powers of $\lambda$ for the $\Lambda$ iry function and its derivative separately. The general equations are thus obtained as

$$
\begin{aligned}
i\left(\nabla \theta \cdot \mathbf{Q}_{(k)}+\zeta \nabla \zeta \cdot \mathbf{R}_{(k)}\right)+ & \nabla \cdot \mathbf{Q}_{(k-1)}-\mathbf{a}_{3}\left(2 \zeta \nabla \theta \cdot \nabla \zeta R_{(k-1)}\right. \\
& \left.+\zeta \nabla \zeta \cdot \nabla \zeta Q_{(k-1)}+\nabla \theta \cdot \nabla \theta Q_{(k-1)}\right)+i \mathbf{a}_{3}\left(\Delta \theta Q_{(k-2)}\right. \\
& +2 \nabla \theta \cdot \nabla Q_{(k-2)}+\nabla \zeta \cdot \nabla \zeta R_{(k-2)}+\zeta \nabla \zeta R_{(k-2)} \\
& \left.+2 \zeta \nabla \zeta \cdot \nabla R_{(k-2)}\right)-i\left(Q_{(k-2)} \nabla \theta \cdot \mathbf{b}+R_{(k-2)} \zeta \nabla \zeta \cdot \mathbf{b}\right) \\
& +\triangle Q_{(k-3)} \mathbf{a}_{3}-\nabla Q_{(k-3)} \cdot \mathbf{b}=0,
\end{aligned}
$$




$$
\begin{aligned}
i\left(\nabla \theta \cdot \mathbf{R}_{(k)}+\nabla \zeta \cdot \mathbf{Q}_{(k)}\right)+ & \nabla \cdot \mathbf{R}_{(k-1)}-\mathrm{a}_{3}\left(2 \nabla \theta \cdot \nabla \zeta Q_{(k-1)}\right. \\
& \left.+\zeta \nabla \zeta \cdot \nabla \zeta R_{(k-1)}+\nabla \theta \cdot \nabla \theta R_{(k-1)}\right)+i \mathbf{a}_{3}\left(\Delta \theta R_{(k-2)}\right. \\
& +2 \nabla \theta \cdot \nabla R_{(k-2)}+\Delta \zeta Q_{(k-2)}+2 \nabla \zeta \cdot \nabla Q_{(k-2)} \\
& -i\left(Q_{(k-2)} \nabla \zeta \cdot \mathrm{b}+R_{(k-2)} \nabla \theta \cdot \mathrm{b}\right) \\
& +\Delta R_{(k-3)} \mathbf{a}_{3}-\nabla R_{(k-3)} \cdot \mathrm{b}=0,
\end{aligned}
$$

where $\mathbf{Q}_{(i)}=\mathbf{R}_{(i)}=\mathbf{0}$ for all $j<0$. This system of equations may now be solved sequentially following the same techniques used for the exponential expansion.

Preliminary solution. It is convenient to introduce the notations

$$
\widehat{\nabla \zeta}=\zeta^{1 / 2} \nabla \zeta
$$

and

$$
R_{(k)}=\zeta^{1 / 2} R_{(k)},
$$

so that for an index value of zero Eqs. (3.4) and (3.5) acquire the symmetric form

$$
\begin{aligned}
& \widehat{\nabla \zeta} \cdot \mathbf{Q}_{(0)}+\nabla \theta \cdot \hat{\mathbf{R}}_{(0)}=0, \\
& \nabla \theta \cdot \mathbf{Q}_{(0)}+\widehat{\nabla} \zeta \cdot \hat{\mathbf{R}}_{(0)}=0 .
\end{aligned}
$$

A solution is obtained by adding and subtracting the equations, and collecting terms to yield the result

$$
(\nabla \theta \pm \widehat{\nabla \zeta}) \cdot\left(\mathbf{Q}_{(0)} \pm \hat{\mathbf{R}}_{(0)}\right)=0
$$

Introducing the notation

$$
\begin{gathered}
\boldsymbol{\Phi}_{(k)}^{ \pm}=\mathbf{Q}_{(k)} \pm \hat{\mathbf{R}}_{(k)} \\
\nabla \phi^{ \pm}=\nabla \theta \pm \widehat{\nabla \zeta}
\end{gathered}
$$

we may write Eq. (3.10) as

$$
\nabla \phi^{ \pm} \cdot \boldsymbol{\Phi}_{(0)}^{ \pm}=0 .
$$

This equation now has a form identical with that of Eq. (2.7) and has the solution

$$
\boldsymbol{\Phi}_{(0)}=-\Phi_{(0)} \mathbf{H},
$$

where

$$
\mathbf{H}=\frac{\varepsilon \cdot \nabla \phi \otimes \nabla \phi \cdot \varepsilon}{\nabla \phi \cdot \nabla \phi}
$$

We have dropped the $( \pm)$ for convenience since there is no confusion at this point. It will be seen in a following development that the $( \pm)$ signs have a simple physical interpretation. From Eqs. (3.11) and (3.14), the first transport tensors are now written as

$$
\begin{aligned}
& \mathbf{Q}_{(0)}=-Q_{(0)} \mathbf{H}, \\
& \hat{\mathbf{R}}_{(0)}=-\hat{R}_{(0)} \mathrm{H},
\end{aligned}
$$


Eiconal equation. Here we establish two coupled equations which together play the role of the eiconal equation of the exponential expansion. It is again convenient to separate the equations into normal and tangential parts and to treat each part separately.

Using the previous notation we may combine the normal parts of Eqs. (3.4) and (3.5) for an index value of 1 as

$$
-\mathbf{a}^{\lambda} \cdot \mathbf{\Phi}_{(0)} \cdot \mathbf{b} \cdot \mathbf{a}_{\lambda}+(\widehat{\nabla} \zeta \cdot \widehat{\nabla} \zeta+\nabla \theta \cdot \nabla \theta) \Phi_{(0)} \pm \nabla \theta \cdot \widehat{\nabla \zeta} \Phi_{(0)}=0 .
$$

This equation can be considered as two equations in $\Phi_{(0)}$ and solved to yield

$$
\nabla \theta \cdot \widehat{\nabla \zeta}=0
$$

Using Eq. (3.19), we note the reduction given by $\widehat{\nabla \zeta} \cdot \widehat{\nabla \zeta}+\nabla \theta \cdot \nabla \theta=\nabla \phi \cdot \nabla \phi$. Combining this result with Eq. (3.18) and using Eq. (3.14), the eiconal equation of our Airy function expansion is obtained as

$$
\nabla \phi \cdot \varepsilon \cdot \mathrm{b} \cdot \varepsilon \cdot \nabla \phi+(\nabla \phi \cdot \nabla \phi)^{2}=0
$$

First transport equation. The tangential parts of Eqs. (3.4) and (3.5) for an index value of 1 are now combined to yield the result

$$
\nabla \phi \cdot \mathbf{\Phi}_{(1)}-i \mathrm{v}_{(1)}=0,
$$

where

$$
\mathbf{v}_{(1)}=\left(\left.Q_{(0)}^{\alpha \beta}\right|_{\alpha} \pm\left.\zeta^{1 / 2} R_{(0)}^{\alpha \beta}\right|_{\alpha}\right) \mathbf{a}_{\beta} .
$$

Noting the identity of form with Eq. (2.11), the solution of Eq. (3.21) may be obtained in the same manner as before to yield

$$
\boldsymbol{\Phi}_{(0)}=-\Phi_{(1)} \mathbf{H}+i \mathbf{M} \otimes\left[\mathbf{v}_{(1)}\right] .
$$

We have defined the operator $\mathbf{M}$ such that

$$
\mathbf{M} \otimes[\mathbf{w}]=\frac{\nabla \phi \otimes \mathbf{w}+\mathbf{w} \otimes \nabla \phi-(\nabla \phi \cdot \mathbf{w}) \mathbf{\delta}}{\nabla \phi \cdot \nabla \phi} .
$$

Using Eq. (3.23) and the normal parts of Eqs. (3.4) and (3.5) for an index value of 1 , we may now develop the first transport equation. We note the following relations:

$$
\begin{aligned}
\triangle \phi & =\Delta \theta \pm \zeta^{1 / 2} \triangle \zeta \pm \frac{1}{2} \zeta^{-1 / 2} \nabla \zeta \cdot \nabla \zeta, \\
\nabla \phi \cdot \nabla \zeta & = \pm \zeta^{1 / 2} \nabla \zeta \cdot \nabla \zeta, \\
\nabla \Phi_{(0)} & =\nabla Q_{(0)} \pm \zeta^{1 / 2} \nabla R_{(0)} \pm \frac{1}{2} \zeta^{-1 / 2} R_{(0)} \nabla \zeta .
\end{aligned}
$$

Furthermore, we define the vector $\hat{\mathbf{X}}$ with components $\hat{X}_{\beta}$ given by

$$
\hat{X}_{\beta}=a^{\lambda} \cdot M \otimes\left[a_{\beta}\right] \cdot b \cdot a_{\lambda} .
$$

From Eqs. (3.16) and (3.17), the components of $\mathbf{Q}_{(0)}$ and $\hat{\mathbf{R}}_{(0)}$ are obtained as

$$
Q_{(0)}^{\alpha \beta}=-Q_{(0)} H^{\alpha \beta}, \quad \hat{R}_{(0)}^{\alpha \beta}=-\hat{R}_{(0)} H^{\alpha \beta} .
$$

Combining these results and using the eiconal equation, we obtain the final result that $2 \nabla \Phi_{(0)} \cdot \nabla \phi+\Phi_{(0)} \Delta \phi-\nabla \Phi_{(0)} \cdot \mathbf{H} \cdot \hat{\mathbf{X}}-\Phi_{(0)} \nabla \cdot \mathbf{H} \cdot \hat{\mathbf{X}}$

$$
\mp 1 / 2 i \zeta^{-1 / 2} \Phi_{(0)} \nabla \zeta \cdot \nabla \zeta=0 .
$$


Eq. (3.28) is the first transport equation for the Airy function expansion. The equation is identical in form to the first transport equation of the exponential expansion except for the last term. As in Ludwig's equation, it is this term which makes the coefficients of the transport function bounded at the caustic. Solving this equation for $\Phi_{(0)}$, we then have the solution for the first terms of the expansion, since $\Phi_{(0)}^{ \pm}=Q_{(0)} \pm$ $R_{(0)}$. Thus, the traces of the first transport tensors are given by the relations

$$
Q_{(0)}=1 / 2\left(\Phi_{(0)}^{+}+\Phi_{(0)}^{-}\right), \quad \hat{R}_{(0)}=1 / 2\left(\Phi_{(0)}^{+}-\Phi_{(0)}^{-}\right)
$$

Since $\Phi_{(0)}^{ \pm}$is solved in terms of $\phi^{ \pm}$, the association of the $( \pm)$is direct.

General transport equation. The general transport equation is obtained using a technique similar to that used for the exponential expansion. Thus, the tangential parts of Eqs. (3.4) and (3.5) may be combined to give the result

$$
\nabla \boldsymbol{\phi} \cdot \boldsymbol{\Phi}_{(k)}-i \mathbf{v}_{(k)}=0
$$

where we have defined

$$
\mathbf{v}_{(k)}=\left(\left.Q_{(k-1)}^{\alpha \beta}\right|_{\alpha} \pm\left.\zeta^{1 / 2} R_{(k-1)}^{\alpha \beta}\right|_{\alpha}\right) a_{\beta}-i \Phi_{(k-2)} \nabla \phi \cdot b-\nabla \Phi_{(k-3)} \cdot \mathbf{b} .
$$

We define the auxiliary tensor $W_{(\lambda)}$ such that

$$
W_{(\lambda)}=W_{(\lambda)}^{\alpha \beta} a_{\alpha} \otimes a_{\beta},
$$

where $W_{(\lambda)}^{\alpha \beta}=\mathbf{a}^{\alpha} \cdot \mathbf{M} \otimes\left[\mathbf{v}_{(\lambda)}\right] \cdot \mathbf{a}^{\beta}$. The components of $\mathbf{W}_{(\lambda)}$ in general include all of the previous transport functions. Then, by solving Eq. (3.30) as before and using the above definitions, the general transport equation of the Airy function expansion becomes

$$
\begin{aligned}
2 \nabla \Phi_{(k)} \cdot \nabla \varphi+\Phi_{(k)} \Delta \varphi-\Phi_{(k)} \nabla \cdot \mathrm{H} \cdot \hat{\mathrm{X}} & -\nabla \Phi_{(k)} \cdot \mathbf{H} \cdot \hat{\mathrm{X}} \mp 1 / 2 i \zeta^{-1 / 2} \nabla \zeta \cdot \nabla \zeta \Phi_{(k)} \\
& +\nabla \cdot \mathbf{W}_{(k)} \cdot \hat{\mathbf{X}}+\Delta Q_{(k-1)} \pm \Delta R_{(k-1)} \\
& -i \Phi_{(k-2)} \nabla \varphi \cdot \mathbf{b} \cdot \hat{\mathbf{X}}-\nabla \Phi_{(k-3)} \cdot \mathbf{b} \cdot \hat{\mathbf{X}}=0 .
\end{aligned}
$$

As before, this equation is valid for all $k$ if terms with a negative index are dropped. The complete solution may now be written in terms of the solution of the eiconal equation.

Solution of transport equations. By defining functions in a similar fashion to the previous solutions for the exponential expansion, we can obtain solutions for the transport equations in terms of integrals involving the eiconal function. The solution for the first transport function follows from Eq. (3.28) by noting that the characteristics of the eiconal equation have directions given by the vector

$$
\hat{\mathbf{\Lambda}}=2 \nabla \phi-\mathbf{H} \cdot \hat{\mathbf{X}} \text {. }
$$

By defining

$$
\hat{\Gamma}=\left(\Delta \phi+\nabla \cdot \mathbf{H} \cdot \hat{\mathbf{X}} \mp 1 / 2 i \zeta^{-1 / 2} \nabla \zeta \cdot \nabla \zeta\right)
$$

the first transport equation can be written as

$$
\nabla \Phi_{(0)} \cdot \hat{\boldsymbol{\Lambda}}+\Phi_{(0)} \hat{\Gamma}=0 .
$$

By using the characteristic lines and their orthogonal trajectories as coordinates, Eq. (3.35) reduces to the ordinary differential equation

$$
\left(d \Phi_{(0)} / d \hat{\sigma}_{1}\right)+\Phi_{(0)}(\hat{\Gamma} / \hat{\Lambda})=0
$$


where $\hat{\sigma}_{1}$ is the characteristic coordinate parameter. We now define the function

$$
\hat{\gamma}=\int^{\dot{\sigma}_{1}} \frac{\hat{\Gamma}}{\hat{\Lambda}} d \hat{\sigma}_{1} \text {. }
$$

The solution for the trace of the first transport tensor is then given by

$$
\Phi_{(0)}=\Phi e^{-\hat{\gamma}},
$$

where $\Phi$ is an arbitrary integration factor.

We proceed as before to develop the solution of the general transport equation. Thus, along the characteristics, the solution of Eq. (3.33) is obtained as

$$
\Phi_{(k)}=e^{-\hat{\gamma}} \int^{\dot{\sigma}_{1}} \hat{\Omega}_{(k)} e^{\hat{\gamma}} d \hat{\sigma}_{1},
$$

where

$$
\begin{aligned}
\hat{\Omega}_{(k)}=-\left(\nabla \cdot \mathrm{W}_{(k)} \cdot \hat{\mathbf{X}}+\Delta Q_{(k-1)}\right. & \pm \Delta R_{(k-1)} \\
& \left.-i \Phi_{(k-2)} \nabla \phi \cdot \mathbf{b} \cdot \hat{\mathbf{X}}-\nabla \Phi_{(k-3)} \cdot \mathbf{b} \cdot \hat{\mathbf{X}}\right) / \hat{\Lambda} .
\end{aligned}
$$

The equation

$$
\Phi_{(k)}=-\Phi_{(k)} \mathrm{H}+i \mathbf{M} \otimes\left[\mathbf{v}_{(k)}\right]
$$

is obtained finally as the solution for the general transport tensor of the Airy function expansion. The homogeneous solution of Eq. (3.33) is contained in Eq. (3.37) and so is not included in Eq. (3.38).

The solution can be written in terms of previous transport functions by defining the operator $\hat{M}$ so that $\hat{\Omega}_{(k)}=\hat{M}\left[\Phi_{(k)}\right]$, where

$$
\hat{M}\left[\Phi_{(\lambda)}\right]=\left(\nabla \cdot \mathrm{W}_{(\lambda)} \cdot \hat{\mathrm{X}}+\Delta Q_{(\lambda-1)} \pm \Delta R_{(\lambda-1)}-i \Phi_{(\lambda-2)} \nabla \phi \cdot \mathrm{b} \cdot \hat{\mathrm{X}}\right) / \hat{\Lambda} .
$$

We then obtain the solution

$$
\Phi_{(k)}=e^{-\hat{\gamma}} \int^{\hat{\sigma}_{1}} \hat{M}\left[\Phi_{(k-1)}\right] e^{\hat{\gamma}} d \hat{\sigma}_{1} \quad(k \geq 1) .
$$

The entire solution of the problem is now determined a series of integrals involving the eiconal function. This solution may be completely related to the solution of our previous exponential expansion by use of appropriate asymptotic expansions of the Airy functions.

Solution in terms of exponential solution. The similarity of form of the equations obtained from the exponential expansion and those obtained from the Airy function expansion leads to a consideration of the reduction of the one solution to the other. If we set $i \nabla \xi^{ \pm}=\nabla \phi^{ \pm}$, the eiconal equations of the exponential and Airy expansions are identical. Thus, we have the relation

$$
i \xi^{ \pm}=\theta \pm \frac{2}{3} \zeta^{3 / 2},
$$

so that

$$
\theta=i\left(\xi^{+}+\xi^{-}\right) / 2, \quad \frac{2}{3} \zeta^{3 / 2}=i\left(\xi^{+}-\xi^{-}\right) / 2 .
$$

At the caustic, $\xi^{+}=\xi^{-}$so that $\zeta=0$ and the proper behavior for the Airy function is assured. 
Considering Eq. (3.1), we have for sufficiently large values of the Airy function argument

$$
\hat{\mathbf{P}} \sim \frac{\exp (-i \pi / 4)}{2 \pi^{1 / 2} \lambda^{1 / 6}}\left(\mathbf{P}^{+} \exp \left(i \lambda \xi^{+}\right)+\mathbf{P}^{-} \exp \left(i \lambda \xi^{-}+i \pi / 2\right)\right)
$$

where

$$
(\mathbf{Q} \pm \hat{\mathbf{R}})=\zeta^{1 / 4} \mathbf{P}^{ \pm}=\boldsymbol{\Phi}^{ \pm}
$$

Eq. (3.43) is valid at sufficiently large distances from the caustic. For solution of the wave equation, the additional factor $\pi / 2$ in the last term is interpreted as a phase shift as light passes through the caustic. In our case, it is absorbed into the arbitrary integration factor.

We now have the complete solution of the Airy function expansion in terms of the previous exponential expansion. For caustics of the type shown in Fig. 1, Eq. (3.43) is interpreted in the usual sense of the exponential solution along two different characteristics. Thus, each point on the convex side of the caustic is intersected by two characteristics, one of which is incoming to the point from the caustic, the other of which is outgoing from the point to the caustic. The signs of the eiconal function may be identified as illustrated, and will give corresponding signs to the transport function. For the Airy function solution, we first solve the eiconal and transport equations for the exponential expansion and then make the identifications above to yield the complete asymptotic solution.

Near the caustic, the contribution of the term involving the derivative of the Airy function becomes small, and the solution may be simplified. Thus, near the caustic we
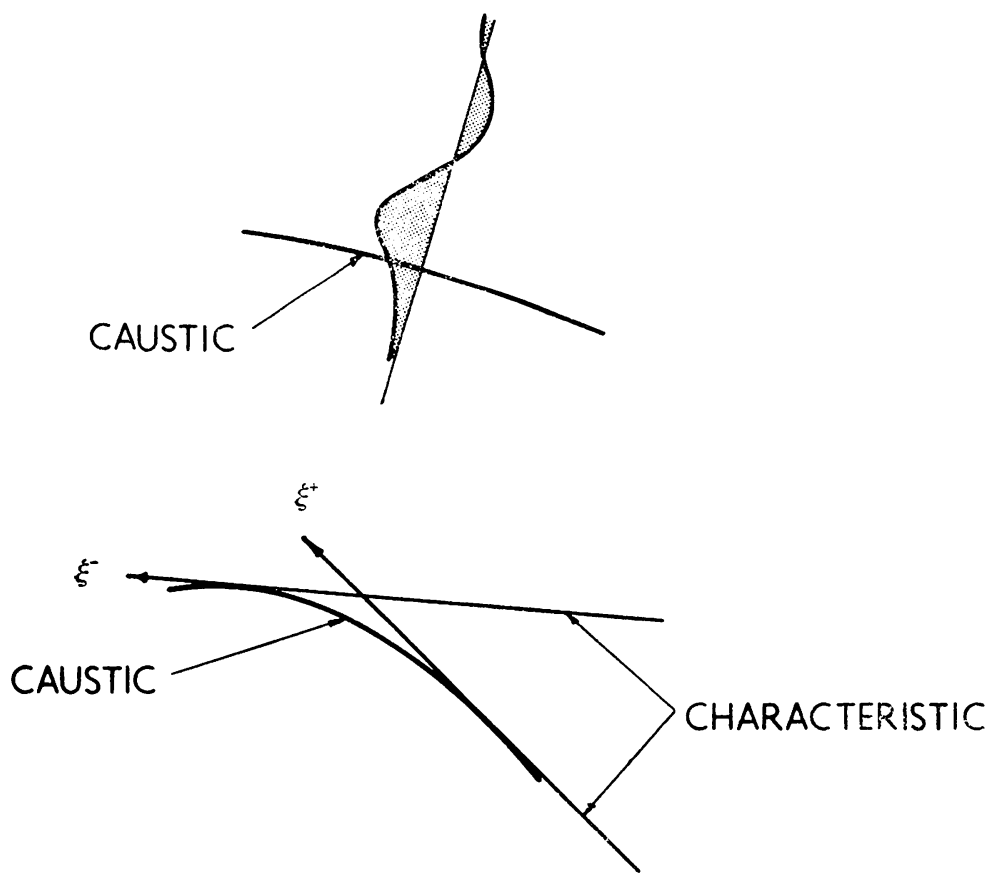

Fig. 1. Solution at a convex caustic. 
have the relations $\left|A_{i}(t)\right| /\left|A_{i}^{\prime}(t)\right|=O(1)$ and $\left|\lambda^{-1 / 3}\right|=O\left(c^{1 / 6}\right)$. For sufficiently small $c$, we may then approximate the solution Eq. (3.43) by the result

$$
\hat{\mathbf{P}} \sim \exp (i \lambda \theta) A_{i}\left(-\lambda^{2 / 3} \zeta\right) \mathbf{Q} .
$$

At sufficiently large distances away from the caustic we have that $\left|A_{i}(t)\right| /\left|A_{i}^{\prime}(t)\right|=$ $O\left(t^{-1 / 2}\right)$. If the argument of the Airy function satisfies the requirement $\zeta \sim \lambda^{-2 / 3}$, the contribution of the term containing the derivative of the Airy function is as large as that due to the Airy function itself, leading to Eq. 3.43 on the convex side of the caustic. Thus, Eq. 3.45 is valid inside a boundary layer of width $O\left(c^{1 / 3}\right)$ about the caustic, illustrated in Fig. 2. Eq. 3.43 is valid outside the layer on the convex side of the caustic.

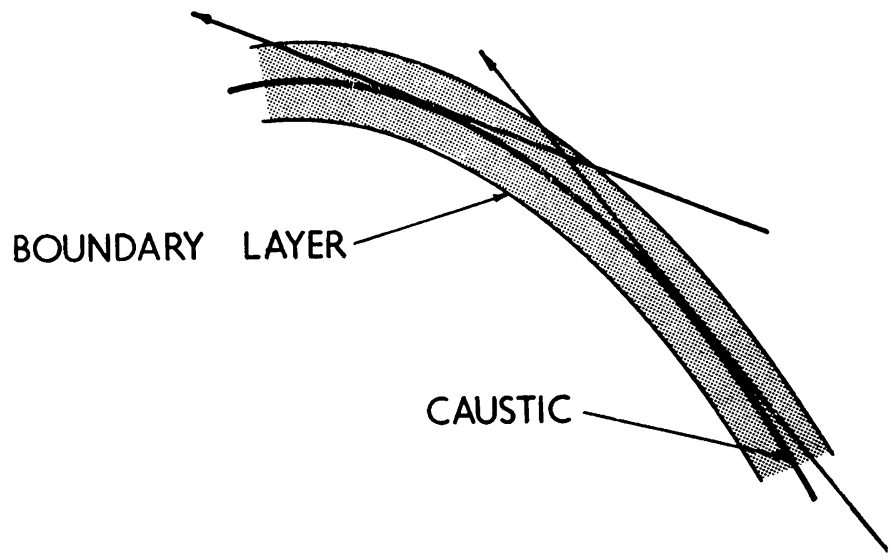

Fig. 2. Boundary layer at a caustic.

Considering the asymptotic form of the Airy functions used to obtain this result, we note that $\left|\arg \left(-\lambda^{2 / 3} \zeta\right)\right|<2 \pi / 3$, so that our expansion in terms of the complex argument is valid.

Complex characteristics. We now consider the concave side of the caustic and introduce complex characteristics, following Keller [23]. Then, considering the asymptotic expansion of the Airy functions for positive argument, we obtain the result

$$
\hat{\mathbf{P}} \sim \frac{1}{2 \pi^{1 / 2} \lambda^{1 / 6}} \exp (i \lambda \bar{\phi}) \overline{\mathbf{\Phi}} .
$$

We have defined a complex eiconal function $\bar{\phi}=\theta+i \psi$, where $\psi=\frac{2}{3}(-\zeta)^{3 / 2}$, and a complex amplitude tensor

$$
\overline{\mathbf{\Phi}}=(-\zeta)^{-1 / 4}\left[\mathrm{Q}-i(-\zeta)^{1 / 2} \mathbf{R}\right] .
$$

The functions corresponding to $\bar{\phi}=\theta-i \psi$ yeild a solution that increases exponentially with increasing $(-\zeta)$, and has been deleted to satisfy the physical requirements of our problem.

To establish the validity of the asymptotic expansion of the Airy function, we note that $\left|\arg \left(\lambda^{2 / 3} \zeta\right)\right|<\pi$, so that our expansion is valid.

There remains the case where a third characteristic can intersect points on the convex side of the caustic, as illustrated in Fig. 2. This case is covered directly by the previous exponential expansion since the caustic shown does not influence the solution on this 
third characteristic. Thus, the complete solution will be a linear superposition of the previous solutions and this third solution.

IV. Solution of special cases. In this section we consider explicit solutions of the previous equations. Although it is unlikely that the general integrals developed in the previous sections can be evaluated in closed form for the arbitrary shell, useful insight can be obtained by consideration of elementary cases.

Eiconal function for circular cylinders. Using as coordinates the generators and the parallel circles of the cylinder, denoted by $s$ and $\theta$ respectively, the eiconal equation for the exponential expansion becomes

$$
-\frac{p^{2}}{R}+\left(p^{2}+\frac{q^{2}}{R^{2}}\right)^{2}=0 .
$$

The initial conditions are given by the relations

$$
\xi(0)=\xi_{0}(t), \quad s(0)=s_{0}(t), \theta(0)=\theta_{0}(t),
$$

where we use the initial circumferential angle as the boundary parameter, $t$. In addition, we require the usual strip conditions. The subscript $(0)$ is used to indicate the initial value.

Eq. (4.1) is of a special form and has the solution

$$
\xi=p_{0}\left(s-s_{0}\right)+q_{0}(\theta-t)+\xi_{0} .
$$

For this special case, the characteristics are straight lines and hence are geodesics of the shell surface. The functions $p_{0}$ and $q_{0}$ are parametric in the boundary parameter $t$, and are obtained by combining the initial and strip conditions. Further solution of the equations requires a specific boundary configuration.

For the case of slowly varying data, the boundary curve is a level line on the eiconal surface. Therefore, the gradients of the eiconal function are normal to the boundary, but are not straight lines. In the geometric optics solution of the wave equation for the case of an isotropic medium with a constant refractive index, the characteristics and gradients are coincident straight lines, and define the rays. In our case, however, the characteristics are not in general coincident with the gradient lines and this lack of coincidence is a principal difficulty in solving the equations in the large.

Cylinder with an oblique edge. An elementary boundary curve is given by the intersection of a plane with the cylinder, illustrated in Fig. 3. The initial conditions become, for the case of slowly varying data,

$$
\xi_{0}=0, \quad s_{0}=R \tan \psi \cos t, \quad \theta_{0}=t .
$$

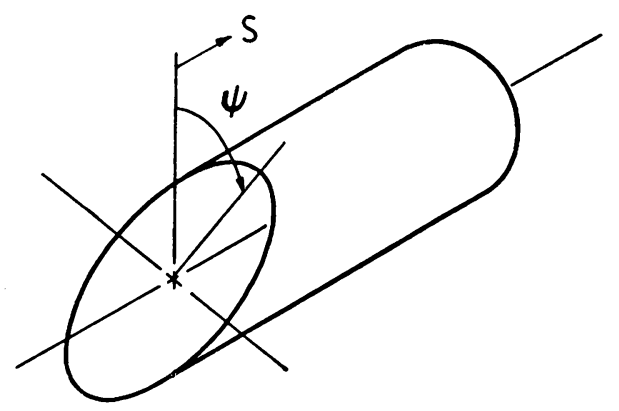

Fig. 3. Cylinder with an oblique edge. 
The eiconal function is obtained as

$$
\xi=\left(1+\tan ^{2} \psi \sin ^{2} t\right)^{-1}\left[R^{-1 / 2}\left(s-s_{0}\right)-R^{1 / 2} \tan \psi \sin t(\theta-t)\right],
$$

and the characteristics are defined by the relation

$$
(\theta-t)=\frac{2}{R}\left(s-s_{0}\right)\left(\frac{\tan \psi \sin t}{1-\tan ^{2} \psi \sin ^{2} t}\right) .
$$

The equation for the caustic is obtained by finding the envelope of the characteristics, and is given parametrically by

$$
\begin{aligned}
\theta & =t-\tan t, \\
\frac{s}{R} & =\cos t \tan \psi-\frac{1}{2}\left(\frac{1-\sin ^{2} t \tan ^{2} \psi}{\cos t \tan \psi}\right) .
\end{aligned}
$$

Fig. 4 illustrates the nature of the characteristics and the caustic near the edge of the developed cylinder.

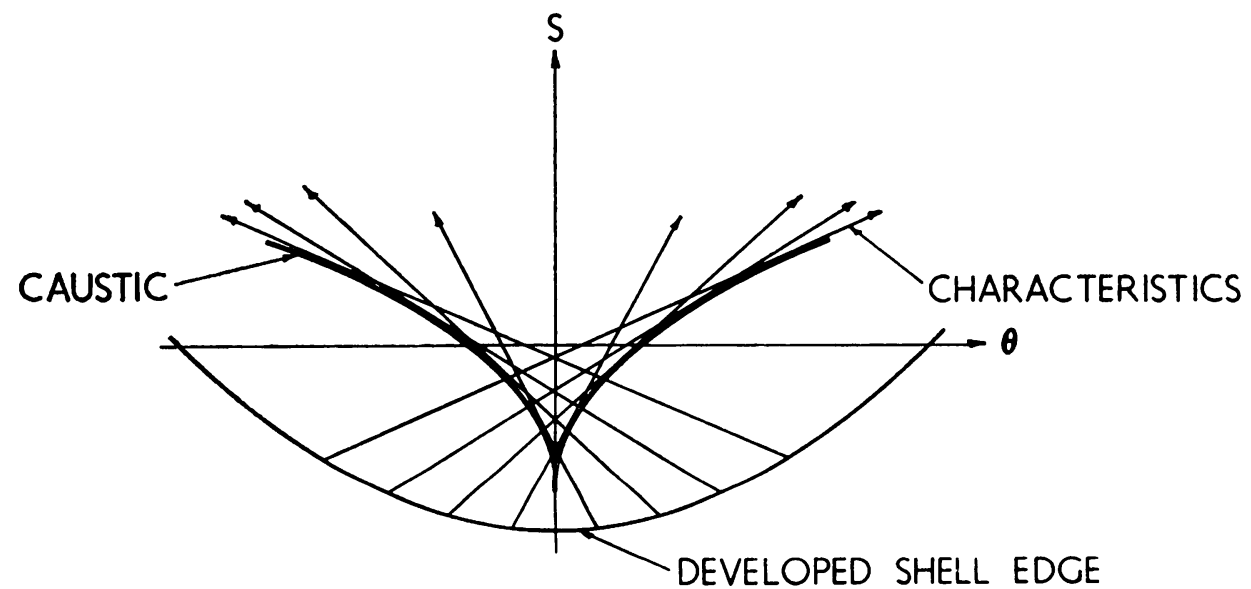

Fig. 4. Caustic for an oblique edge.

We note that the solution obtained is for stresses and deformations that have a decaying behavior into the interior, so that the caustic is, in general, in a region of low stresses and deformations. For example, with $\psi=50^{\circ}, t=\pi$, the ratio of the caustic stress to the edge stress for an aluminum cylinder with $R / h=212$ is $\exp (-21.6)$. As the inclination angle of the edge increases, the caustic moves closer to the edge, as illustrated in Fig. 5. It may be that the effect of the caustic would be important for relatively thick shells with large edge inclination angles, but it appears that there is negligible practical importance.

In addition to the previously discussed stress intensification effect, there is the possibility of the intersection of non-adjacent characteristics in the region interior to the caustic, illustrated in Fig. 4. This intersection provides an additional intensification but the practical significance is negligible in light of the previous comment.

Cylinder with a circular hole cutout. In this section we consider a boundary that is formed by a circular hole in the developed cylinder, illustrated in Fig. 6, and compare 


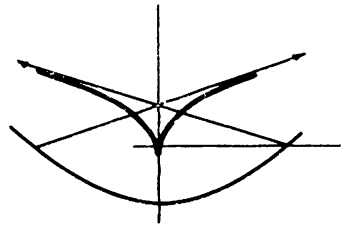

$\psi=30^{\circ}$

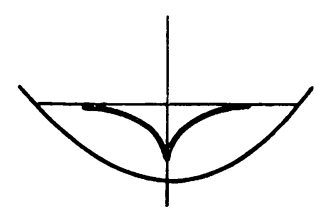

$45^{\circ}$

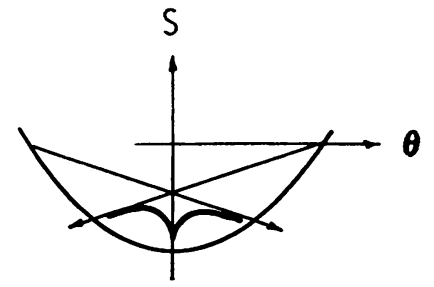

$60^{\circ}$

Fig. 5. Effect of $\psi$ on caustic.

the exponential and Airy function expansions. The special interest in boundaries of this type derives from the singular behavior of solutions at points of tangency of the boundary and asymptotic lines; see, for example, Goldenveizer [3]. For the cylinder, the generators are asymptotic lines, and our solution shows the existence of a caustic at that point of tangency.

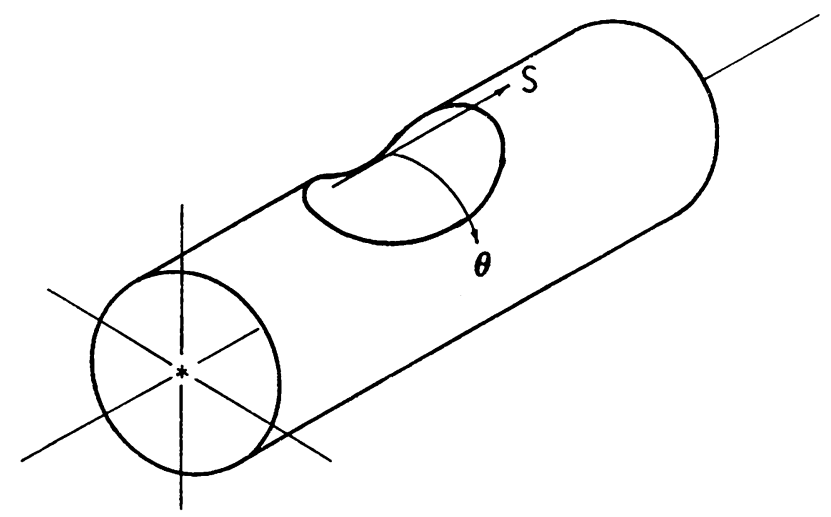

FIG. 6. Cylinder with a circular hole cutout.

Using the coordinates illustrated in Fig. 6, the initial conditions for slowly varying data become

$$
\xi_{0}=0, \quad s_{0}=\left(r_{0}^{2}-R^{2} t^{2}\right)^{1 / 2}, \quad \theta_{0}=t .
$$

The eiconal function is obtained as

$$
\xi=\left(r_{0}^{2}-R^{2} t^{2}\right)^{1 / 2}\left[\left(r_{0}^{2}-R^{2} t^{2}\right)^{1 / 2}\left(s-s_{0}\right) R^{-1 / 2}+R^{3 / 2} t(\theta-t)\right] / r_{0}^{2},
$$

and the characteristics are determined by the equation

$$
(\theta-t)=2 t \frac{\left(r_{0}^{2}-R^{2} t^{2}\right)^{1 / 2}}{\left(r_{0}^{2}-R^{2} t^{2}\right)}\left(s-s_{0}\right) .
$$

The caustic is obtained as the envelope of Eq. (4.10) and is illustrated for the dcveloped hole in Fig. 7. As before, the characteristics are straight lines, but their caustie now forms in the hole itself. We note that only a portion of the shell is affected by the boundary layer, as shown in Fig. 8. We expect our solution to be valid everywhere except perhaps at the point of tangency of the boundary and the asymptotic line. Indeed, 


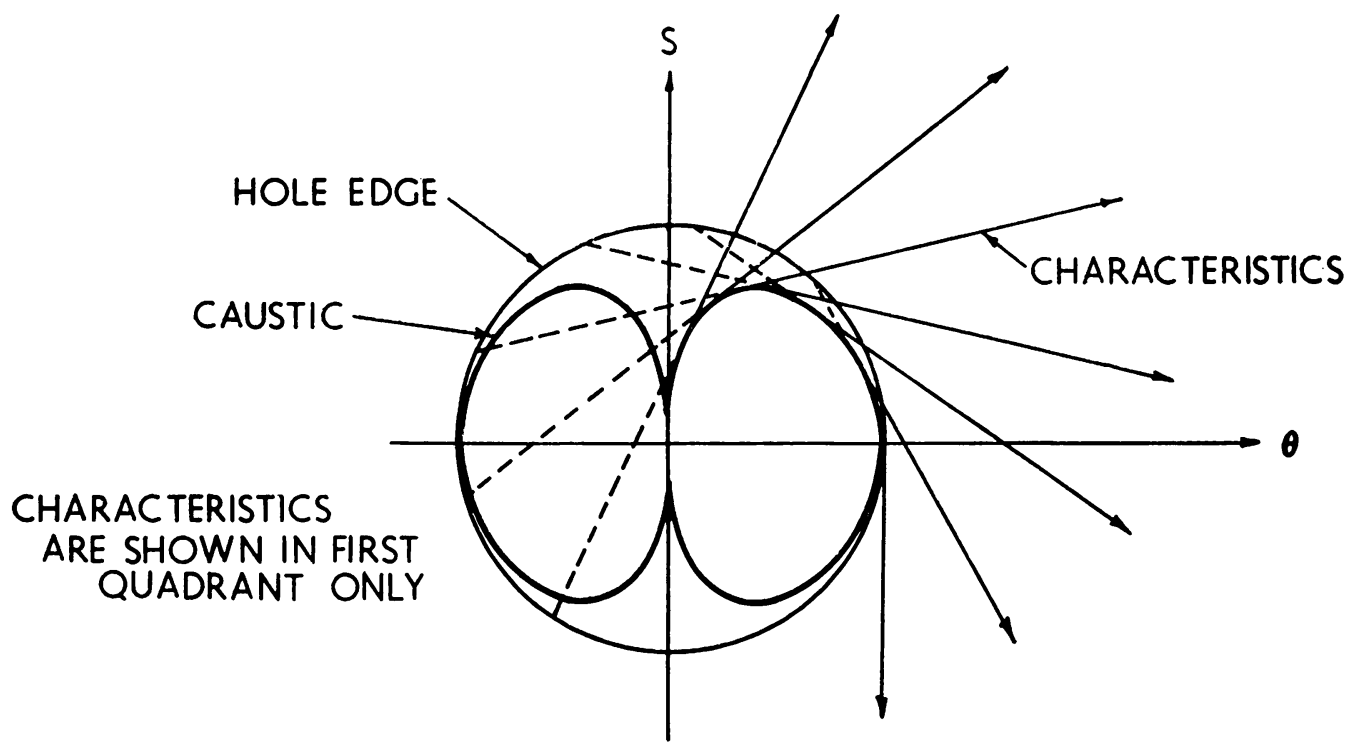

Fig. 7. Caustic for a circular hole cutout.

this is also the point of tangency of the boundary curve and the caustic, and by prior discussion, the equation for the first transport function becomes singular at that point. However, it is shown subsequently that at small distances from the edge our exponential solution is still valid. We note here that the eiconal function is zero at the point of tangency and so it is a regular function and satisfies the initial conditions

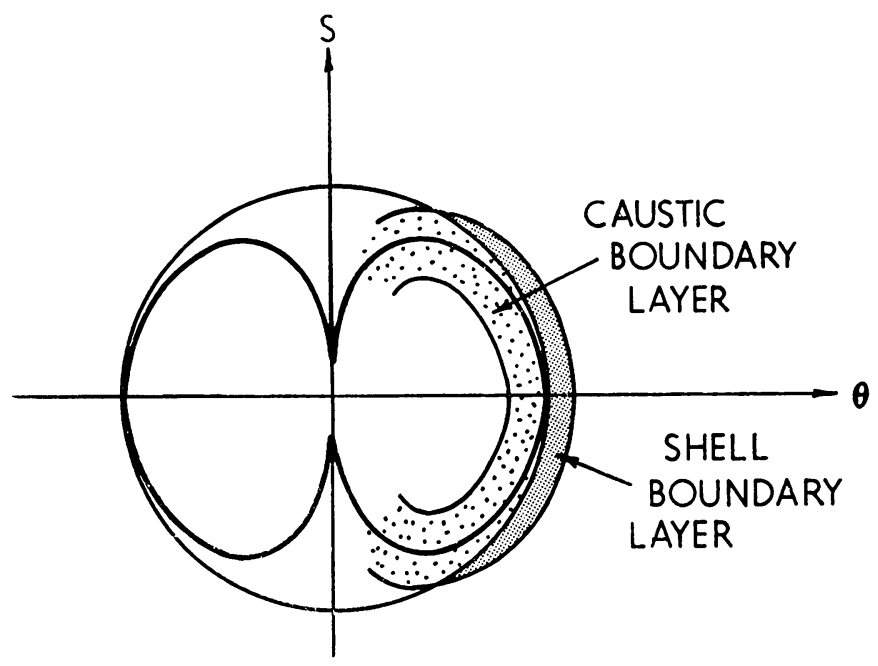

FIG. 8. Boundary layer for circular hole cutout.

Steele [1] solves the problem explicitly for the case in which the hole is a rigid insert with a simply supported boundary. The shell is loaded axially and the boundary conditions for the edge-effect solution are that the mid-surface strain tangent to the boundary is the negative of the membrane strain, and that the bending stresses are zero. 
For this case, we have that $\xi_{0}=0$. In order to solve for the transport functions, the coordinates are now taken as the characteristics and the level lines, or contours, of the eiconal surface, and so represents a non-orthogonal system. Using $\zeta$ as the coordinate parameter along the characteristics, we obtain the solution of the eiconal equation in this new system as

$$
\xi=\cos ^{2} \eta\left[\left(s-r_{0} \cos \eta\right)^{2}+\left(R \theta-r_{0} \sin \eta\right)^{2}\right]^{1 / 2} / R^{1 / 2} .
$$

The first transport function becomes

$$
P_{(0)}=P a^{-1 / 2},
$$

where $P$ is the arbitrary integration factor. The angle $\eta$ is the polar angle, measured from the generator that passes through the hole center. For the cylinder, we obtain the relation

$$
a^{1 / 2}=\left(R^{3 / 2} / \cos \eta\right)\left[1+\left(2 \xi /\left(R^{1 / 2} \cos ^{3} \eta\right)\right)\right] .
$$

It is of some interest at this point to digress and consider cutouts with the boundary curve given by a higher-order curve. For example, cutouts with an aspect ratio of one can be described by $s^{n}+(R t)^{n}=1$. For very large $n$, the sides approach straight lines, and the caustic acquires a higher order of contact with the side that is parallel to the cylinder generators. In the limit of high $n$, our solution, as expected, then loses its exponentially decaying behavior in the circumferential direction, and recovers the ordinary edge-effect solution for cylinders in the axial direction. However, in the circumferential direction, the first transport equation would still have singular coefficients so that our solution is not valid. Goldenveizer [3] solves this problem naming it the "generalized edge effect," in which the entire edge is an asymptotic line.

Returning to the circular cutout with a rigid insert, we now consider the nature of the Airy function solution. From Eq. (4.12) and the solution obtained from the Airy function expansion Eq. (3.44), the trace of the first transport tensor is given by

$$
\Phi_{(0)}^{x}=\zeta^{1 / 4} P_{(0)}^{ \pm} .
$$

The choice of the $( \pm)$ is somewhat arbitrary and the convention has been adopted to take $\xi^{+}$in the direction forward from the caustic when transversing the caustic in the direction of positive $\eta$, and $\xi^{-}$conversely.

For our problem, the solution given by Eq. (4.12) may be written as

$$
P_{(0)}^{ \pm}= \pm P /(a \cos \eta)^{1 / 2} \text {. }
$$

An arbitrary function of the polar angle has been introduced into the solution in order to obtain the desired behavior at $\eta=\pi / 2$. Using Eq. (4.14), we obtain the solution in the boundary layer, illustrated in Fig. 8, from Eq. (3.45) as

$$
\hat{\mathbf{P}} \sim \exp (i \lambda \theta) A_{i}^{\cdot}\left(-\lambda^{2 / 3} \zeta\right) \zeta^{1 / 4}\left(P_{(0)}^{+}+P_{(0)}^{-}\right) \mathrm{H} .
$$

Using Eqs. (4.14) and (4.15), a one-term approximation to the trace of $\mathbf{P}$ is obtained as

$$
\hat{P} \sim P \exp (i \lambda \theta) A_{i}\left(-\lambda^{2 / 3} \zeta\right) \frac{\zeta^{1 / 4}}{a^{1 / 2}}\left(\frac{1}{\cos ^{1 / 2} \eta^{+}}+\frac{1}{\cos ^{1 / 2} \eta^{-}}\right)
$$

By assuming that the limit of Eq. (4.16) at the caustic is approached from the lefthand and right-hand sides at the same rate, $P$ may be shown to be continuous with a 
bounded derivative in the entire boundary layer, including the edge of the hole. Furthermore, we have shown previously that the Airy function solution reduces to the exponential solution outside of the boundary layer. Therefore, the solution represented by Eq. (3.1) is valid in the large for the entire boundary region.

It should be noted that each characteristic intersects the boundary twice, except at $\eta=\pi / 2$, as illustrated in Fig. 7. Due to this effect, there will in general be a violation of the prescribed boundary data. For completeness, a series of auxiliary boundary value problems could then be superimposed to reestablish the correct boundary data. However, since our solution exhibits an exponential decay along the characteristics, the contribution of this effect will be small except in the boundary layer itself, where it is this additional intersection that gives our solution the desired behavior. In addition, due to the symmetry of the caustic, there are two characteristics from the opposite side of the hole that will also pass through a given point. Again, however, due to the exponential nature of the solution, this contribution will be negligible. We note that at the position $\eta=0$ on the hole edge, the usual edge-effect solution for the cylinder with a symmetric boundary is obtained. However, along the circumferential line from $\eta=\pi / 2$, we have that $\xi^{+}=-\xi^{-}$, so that from Eq. (3.42) $\theta=0$ and the solution displays the expected Airy function behavior that was illustrated in Fig. 1.

In this section we have shown formally that the edge-effect solution represented by Eq. (3.1) is uniformly valid throughout the edge-effect boundary region, including the point of tangency of the boundary and an asymptotic line. It is expected that this uniform validity would hold in general, but we have demonstrated only a simple example, and a general proof has not yet been shown.

Other shells. It was possible to consider the solution for the circular cylinder in some detail due to the simplicity of the eiconal equation. However, for a shell of revolution, we find at once that the eiconal equation becomes sufficiently complicated apparently to preclude a closed-form analytical result, at least at this time. The principal complication is that the radius is no longer constant so that the eiconal equation involves a coordinate parameter explicitly. For example, even for a right circular cone the characteristics are no longer straight lines but are solutions of a nonlinear ordinary differential equation. The behavior of the cone is then somewhat different from the cylinder even though both shells have zero Gaussian curvature.

By considering a shell of revolution with an axisymmetric boundary, we note that the choice of coordinates for solution of the transport functions appears as a natural one. For this special case, the ciconal equation reduces to

$$
(d \xi / d s)^{2}=1 / R_{2}
$$

where $s$ is arc length along the characteristics. The eiconal surface is itself a surface of revolution, and the characteristics thus coincide with the meridians of the shell surface. The level curves of the eiconal surface are projections of the parallel circles of the shell, so that the characteristics and projections of the gradient lines of the eiconal surface also coincide. The complete solution for this case is then easily obtained using the exponential expansion, and is valid everywhere except near the "caustic" which is the point of intersection of the meridians at the top of a closed shell.

V. Concluding remarks. We have obtained a formal solution to the shell equation presented in Sec. I by using a slightly modified form of a generally accepted exponential asymptotic expansion, and the solution is intuitively satisfactory in the sense that it 
yields behavior that may be generally expected. At this point, however, our solution remains a formal one since we have not yet obtained an error estimate, nor have we shown that the solution is actually asymptotic.

The recognition of the similarity of our shell solutions and available asymptotic solutions of the reduced wave equation led to an analogy with geometric optics solutions. The similarity arises due to the role of the Laplacian in corresponding equations and the similarity in the forms of the asymptotic expansions used. There is apparently no advantage in carrying the analogy too far, but it is interesting to note that our shell equation, for the trivial limiting case of a vanishing-curvature tensor, corresponds to the reduced wave equation for the case of zero refractive index. Our principal interest in the geometric optics solutions has been the adoption of some key mathematical details. It should be remarked that our Airy function solution is not expected to be valid in the region of cusps of a caustic. However, Ludwig [22] uses a generalized Airy function for the solution of the reduced wave equation near a cusp, and that type of an expansion should also be successful for our shell equation.

\section{REFERENCES}

[1] C. R. Steele, A geometric optics solution for the thin shell equation, Int. J. Eng. Sci. 9, 681-704 (1971)

[2] T. R. Logan, Asymptotic solutions for shell with general boundary curves. Ph.D. Thesis, Stanford University, July 6, 1970

[3] A. L. Goldenveizer, Theory of elastic thin shells, London, Pergamon Press, 1961

[4] A. van der Neut, Bending at the oblique end section of cylindrical shells, in Proceedings of the Symposium on the Theory of Thin Elastic Shells, W. T. Koiter, ed., Amsterdam, North Holland, 1960, pp. 247-269.

[5] F. Kitching and M. P. Bond, Flexibility and stress factors for mitred bends under in-plane bending, Int. Journal Mech. Sci. 12, 267-285 (1970)

[6] D. E. Johnson, Stresses in a spherical shell with a nonradial nozzle, J. Appl. Mech. 299-307 (1967)

[7] A. K. Naghdi and A. C. Eringen, Stress distribution in a circular cylindrical shell with a circular cutout, Ingenieur-Archiv. 34, 161-172 (1965)

[8] G. N. Savin and A. N. Guz, Stress around curvilinear holes in shells (original in Russian, available in English as National Aeronautical Lab., Bangalore, Report TT-2, Sept. 1967 (NASA No. N6823539))

[9] A. I. Lure, Concentration of stress in the vicinity of an aperture in the surface of a circular cylinder (original in Russian, available in English as New York Univ. Report IMM-NYU 280, Nov., 1960 (AD250308))

[10] Peter Van Dyke, Stresses about a circular hole in a cylindrical shell, A.I.A.A. Journal 3, 1733-1742 (1965)

[11] B. M. N. Rao, and T. Ariman, On the stresses around an elliptic hole in a cylindrical shell, Report UND-70-5, Defense Documentation Center; presented at the Sixth U. S. National Congress of Applied Mechanics, Harvard University. June, 1970

[12] P. M. Naghdi, Foundations of elastic shell theory, in Progress in Solid Mechanics 4, North Holland, 1963, pp. 1-90

[13] P. M. Naghdi, On the differential equations of the linear theory of elastic shells, in Proceedings of the XI International Congress of Applied Mechanics, Munich, 1964, pp. 262-269

[14] V. V. Novozhilov, Thin shell theory, The Netherlands: Noordhoff Ltd., 1964

[15] J. L. Sanders, An improved first approximation theory for thin shells, NASA Report 24, 1959

[16] W. T. Koiter, A consistent first approximation in the general theory of thin elastic shells, in Proceedings of the Symposium on the Theory of Thin Elastic Shells, W. T. Koiter, ed., Amsterdam, North Holland, 1960

[17] Robert M. Lewis and Joseph B. Keller, Asymptotic methods for partial differential equations: the reduced wave equation and Maxwell's equation. New York Univ. Report No. EM-194, 1964

[18] C. R. Steele, An asymptotic fundamental solution of the reduced wave equation on a surface, Q. App. Math. 509-524 (1972) 
[19] Robert M. Lewis et al., Uniform asymptotic theory of creeping waves, Comm. Pure Appl. Math., 20, 295-328 (1967)

[20] C. R. Steele, A systematic analysis for shells of revolution with nonsymmetric loads, Proceedings, 4 th U. S. Nat. Congress of A pp. Mech., pp. 783-792

[21] George B. Airy, On the intensity of light in the neighborhood of a caustic, Trans. Cambridge Philos. Soc. VI, part III, 379-402 and plate 7 (1838)

[22] Donald Ludwig, Uniform asymptotic expansions at a caustic, Comm. Pure $\Lambda$ ppl. Math. 19, 215$250(1968)$

[23] Joseph B. Keller, A geometrical theory of diffraction, in Calculus of variations and its application, New York, McGraw-Hill, 1958, pp. 27-52 